\section{CrossMark} <click for updates

Cite this: Phys. Chem. Chem. Phys., 2015, 17, 4118

Received 2nd October 2014, Accepted 22nd December 2014

DOI: $10.1039 / \mathrm{c} 4 \mathrm{cp} 04461 \mathrm{~d}$

www.rsc.org/pccp

\title{
Charge transfer tuning by chemical substitution and uniaxial pressure in the organic complex tetramethoxypyrene-tetracyanoquinodimethane $\dagger$
}

\author{
Milan Rudloff, ${ }^{a}$ Kai Ackermann, ${ }^{a}$ Michael Huth, ${ }^{\star a}$ Harald O. Jeschke, ${ }^{b}$ Milan Tomic, ${ }^{b}$ \\ Roser Valenti, ${ }^{b}$ Benedikt Wolfram, ${ }^{c}$ Martin Bröring, ${ }^{c}$ Michael Bolte, ${ }^{d}$ \\ Dennis Chercka, ${ }^{e}$ Martin Baumgarten ${ }^{e}$ and Klaus Müllen ${ }^{e}$
}

\begin{abstract}
In the search for novel organic charge transfer salts with variable degrees of charge transfer we have studied the effects of two modifications of the recently synthesized donor-acceptor system [tetramethoxypyrene (TMP)]-[tetracyanoquinodimethane (TCNQ)]. One is of chemical nature by substituting the acceptor TCNQ molecules by $\mathrm{F}_{4} \mathrm{TCNQ}$ molecules. The second consists in simulating the application of uniaxial pressure along the stacking axis of the system. In order to test the chemical substitution, we have grown single crystals of the $\mathrm{TMP}-\mathrm{F}_{4} \mathrm{TCNQ}$ complex and analyzed its electronic structure via electronic transport measurements, ab initio density functional theory (DFT) calculations and UV/VIS/IR absorption spectroscopy. This system shows an almost ideal geometrical overlap of nearly planar molecules stacked alternately (mixed stack) and this arrangement is echoed by a semiconductor-like transport behavior with an increased conductivity along the stacking direction. This is in contrast to TMP-TCNQ which shows a less pronounced anisotropy and a smaller conductivity response. Our band structure calculations confirm the one-dimensional behavior of $\mathrm{TMP}-\mathrm{F}_{4} \mathrm{TCNQ}$ with pronounced dispersion only along the stacking axis. Infrared measurements illustrating the $\mathrm{C} \equiv \mathrm{N}$ vibration frequency shift in $\mathrm{F}_{4} T C N Q$ suggest however no improvement in the degree of charge transfer in $T M P-F_{4} T C N Q$ with respect to TMP-TCNQ. In both complexes about $0.1 \mathrm{e}$ is transferred from TMP to the acceptor. Concerning the pressure effect, our DFT calculations on the designed TMP-TCNQ and TMP- $\mathrm{F}_{4} \mathrm{TCNQ}$ structures under different pressure conditions show that application of uniaxial pressure along the stacking axis of TMP-TCNQ may be the route to follow in order to obtain a much more pronounced charge transfer.
\end{abstract}

\section{Introduction}

Organic charge transfer (CT) systems offer both a playground for studying fundamental solid state properties and a material class that in recent years has become extremely attractive for modern organic electronic devices, such as organic thin film transistors. ${ }^{1-6}$ The electrostatic bonding of two different organic molecules, donor (D) and acceptor (A), gives rise to a rich electronic behavior. This feature combined with the vast

\footnotetext{
${ }^{a}$ Goethe-Universität, Physikalisches Institut, Frankfurt am Main, Germany. E-mail: michael.huth@physik.uni-frankfurt.de

${ }^{b}$ Goethe-Universität, Institut für Theoretische Physik, Frankfurt am Main, Germany

${ }^{c}$ Technische Universität Carolo Wilhelmina, Institut für Anorganische und Analytische Chemie, Braunschweig, Germany

${ }^{d}$ Goethe-Universität, Institut für Anorganische und Analytische Chemie,

Frankfurt am Main, Germany

${ }^{e}$ Max-Planck-Institut für Polymerforschung, Mainz, Germany

$\dagger$ CCDC 1002047. For crystallographic data in CIF or other electronic format see DOI: $10.1039 / \mathrm{c} 4 \mathrm{cp} 04461 \mathrm{~d}$
}

flexibility chemists have concerning molecular design makes it possible to gain access to such phenomena as unconventional superconductivity, ferroelectricity, spin liquid or metal-toinsulator transitions, to mention a few. ${ }^{7-9}$ Charge transfer compounds tend to form one- or two-dimensional structures (stacks or layers) that lead to a simple differentiation between systems composed of segregated stacks of the donor and acceptor and systems where the donor and acceptor are mixed within the stacks. The vast majority of CT compounds are of the latter mixed-stack type and have long been considered as a material class of reduced significance in basic and applied research, as the geometrical arrangement and the resulting molecular orbital overlap lead to semiconducting or insulating behavior. However, this view needs revision in several respects. Ambipolar charge-transport properties have been predicted by electronic structure calculations in selected mixed-stack systems with mobility values that would rival the best single-component organic semiconductors, such as rubrene or pentacene. ${ }^{10,11}$ Moreover, a growing group of mixed-stack CT systems shows 
a temperature- and/or pressure driven transition into a ferroelectric or an antiferroelectric state associated with a pronounced increase of the charge transfer degree, the so-called neutral-ionic (NI) transition. ${ }^{12}$ The NI-transition is widely tunable by pressure (in single crystals) ${ }^{13}$ or biaxial strain (in clamped thin films $)^{14-16}$ and may even be associated with a multiferroic state in which a, typically, antiferromagnetic order parameter couples to the electrical polarization. ${ }^{17}$ With a view on strengthening the material basis for this class of mixedstack systems, several challenges have to be overcome. Despite the fact that most of the organic CT systems crystallize in a mixed-stack variant, the conditions for the establishment of a temperature-driven NI-transition are rather stringent. Roughly speaking, starting from a moderate initial charge transfer degree of about 0.3 to 0.4 from the donor to the acceptor the gain of Madelung energy under thermal contraction as the material cools down must be sufficient to overcompensate the generally still positive difference in the ionization energy of the donor and the electron affinity of the acceptor. Under these conditions a spontaneous increase of the charge transfer degree, usually in conjunction with D-A dimerization and a loss of inversion symmetry, can occur leading to an (anti-) ferroelectric state. At the beginning of a systematic identification of possible candidates for a NI-transition lies the proper selection of the donor and acceptor species based on their respective molecular energies, as has been suggested by Torrance (V-shaped diagram).$^{18}$ It has to be noted, though, that a good match of the molecular energies does not guarantee a NI-transition to occur. By way of proper modification of the functional groups of either the donor or the acceptor fine-tuning of the respective ionization energies and electron affinities can be done. However, in co-crystallizing the donor and acceptor a possible change of the resulting crystal structure as compared to the mother compound is hard to prevent and not predictable. Pressure-based tuning of the mother compound, ideally leaving the crystal structure unchanged, is an attractive alternative, albeit technically nontrivial and thus not suitable as a screening method for the identification of new NI-transition systems. Here the question arises as to which approaches with anticipatory quality can be developed that allow one to identify possible candidates beforehand, once a promising mother compound is identified.

In this work we present a combined experimental and theoretical study on the electronic properties of the new mixed-stack organic CT compound [tetramethoxypyrene (TMP)]-[tetrafluorotetracyanoquinodimethane $\left.\left(\mathrm{F}_{4} \mathrm{TCNQ}\right)\right]$ which was derived from the previously synthesized TMP-TCNQ mixed-stack system by way of enhancing the acceptor qualities of TCNQ by halogen substitution. The pyrene TMP and the TCNQ structure (see Fig. 1) both offer a multitude of possible functional modifications that allow one to investigate how specific changes affect the structural and electronic properties of the CT compound. TMP-F 4 TCNQ has a triclinic crystal structure with virtually ideal geometrical overlap of the donor and acceptor resulting in a pronounced anisotropy of the electronic properties, as deduced from temperaturedependent conductivity measurements and density functional theory calculations. This structure differs from that of the mother
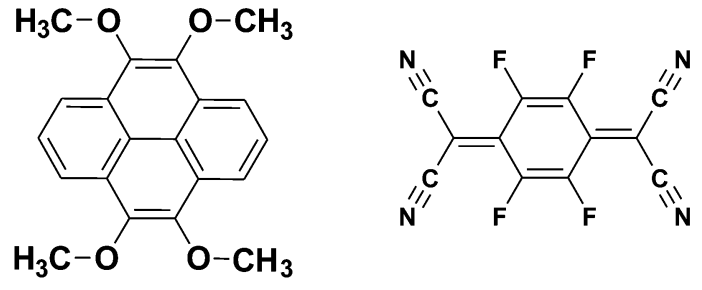

Fig. 1 Molecular structure of TMP (left) and $\mathrm{F}_{4} \mathrm{TCNQ}$ (right). The four methoxy groups provide TMP with donor properties while the four cyano groups combined with four fluorine atoms give $\mathrm{F}_{4} \mathrm{TCNQ}$ its acceptor functionality.

compound TMP-TCNQ which is far less anisotropic. Somewhat counter-intuitively, the degree of charge transfer of TMP- $\mathrm{F}_{4} \mathrm{TCNQ}$ is not enhanced as compared to that of TMP-TCNQ but even reduced from about 0.2 to 0.1 , according to the observed shifts in the $\mathrm{C} \equiv \mathrm{N}$ stretching mode as seen in IR absorption spectroscopy as well as in the density functional theory (DFT) calculations. By analyzing the charge distribution of the two complexes, we propose a possible explanation of these observations. Finally by simulating the effect of uniaxial pressure on these two compounds within the DFT calculations, we predict a pronounced increase of the charge transfer degree for TMP-TCNQ whereas TMP-F 4 TCNQ appears to be mostly unaffected. This band-structure-based approach of a simulated pressure experiment may represent an efficient strategy to identify possible candidates for NI-transition systems based on newly synthesized mixed-stack organic charge transfer systems of suitable initial charge transfer degree.

\section{Methods}

\subsection{Experimental methods}

2.1.1 Synthesis and structure determination. TMP was synthesized at the Max Planck Institute for Polymer Research with a purity of better than $95 \%$. $\mathrm{F}_{4}$ TCNQ was purchased from Sigma-Aldrich with a purity of $\geq 97 \%$.

Single crystals of TMP-F 4 TCNQ were grown in solution by slow evaporation of the solvent. TMP and $\mathrm{F}_{4}$ TCNQ were separately solubilized in dichloromethane (DCM) in equimolar amounts (ca. $10 \mathrm{mg}$ ). Both solutions were carefully mixed in a glass beaker which was then covered with aluminum foil to limit the evaporation rate. Black, needle-like crystals could be extracted after one to two weeks.

The structure determination was based on a $0.41 \times 0.06 \times$ $0.06 \mathrm{~mm}$ sized crystal. X-ray data were recorded at $T=173 \mathrm{~K}$ on a STOE IPDS II two-circle-diffractometer using $\mathrm{Mo}^{-\mathrm{K}_{\alpha}}$ radiation and a Genix 3D multilayer optics monochromator. SHELXS97 $7^{19}$ was used for structure solution while refinement was carried out with SHELXL97. Further information on data collection and structure refinement is given in Table 1. Crystallographic data for $\mathrm{TMP}-\mathrm{F}_{4} \mathrm{TCNQ}$ have been deposited with the Cambridge Crystallographic Data Centre. ${ }^{20}$

Computer programs: X-AREA (Stoe \& Cie, 2001), SHELXS97 (Sheldrick, 2008), SHELXL97 (Sheldrick, 2008), XP in SHELXTLPlus (Sheldrick, 2008). 
Table 1 Experimental details and crystal structure data for $T M P-F_{4} T C N Q$

Crystal data

Chemical formula

$M_{\mathrm{r}}$

Crystal system, space group

Temperature $(\mathrm{K})$

$a, b, c(\AA)$

$\alpha, \beta, \gamma\left({ }^{\circ}\right)$

$V\left(\AA^{3}\right)$

Radiation type

$\mu\left(\mathrm{mm}^{-1}\right)$

Crystal size (mm)

Data collection

Diffractometer

Absorption correction

$T_{\min }, T_{\max }$

No. of measured, independent and

observed $[I>2 \sigma(I)]$ reflections

$R_{\text {int }}$

$(\sin \theta / \lambda)_{\max }\left(\AA^{-1}\right)$

0.595

Refinement

$R\left[F^{2}>2 \sigma\left(F^{2}\right)\right], \mathrm{w} R\left(F^{2}\right), S$

No. of reflections

No. of parameters

$\mathrm{H}$-atom treatment

$\Delta \rho_{\max }, \Delta \rho_{\min }\left(\mathrm{e} \AA^{-3}\right)$

$0.055,0.138,0.95$

2305

201

$\mathrm{H}$-atom parameters constrained

$0.25,-0.25$

2.1.2 Electrical conductivity measurements. Single crystals were placed on a custom-made carrier chip and electrically contacted with conducting graphite paste. $50 \mu \mathrm{m}$ thick gold wires were attached to the paste and soldered to contact pins on the carrier chip. On the small crystals two-probe measurements proved to be sufficient, because the sample resistance dominated by far the contact and wiring resistances. Low temperature measurements were performed in an Oxford ${ }^{4} \mathrm{He}$ cryostat with a Keithley 2636A two-channel sourcemeter. Within the cryostat the carrier chip was mounted in a variable temperature insert (VTI). The temperature was measured using a calibrated resistance thermometer (Cernox).

2.1.3 Infrared spectroscopy. Infrared spectroscopy was applied to powder material from solution growth in the case of TMP-F $\mathrm{F}_{4}$ TCNQ and the as-supplied powder in the case of $\mathrm{F}_{4} \mathrm{TCNQ}$ as a reference. Spectra were recorded at room temperature using a Bruker Vertek 70 FT-IR spectrometer between 600 and $4000 \mathrm{~cm}^{-1}$. All measurements were performed with a resolution of $2 \mathrm{~cm}^{-1}$.

2.1.4 UV-VIS/IR spectroscopy. Spectra were recorded in solution (DCM) on a Perkin-Elmer Lambda 900 UV/VIS/NIR spectrometer. A concentration of $10^{-4}$ mol $\mathrm{l}^{-1}$ was used for absorption between 0.1 and 1 in the wavelength region of experimental interest.

\subsection{Theoretical methods}

We performed $a b$ initio density functional theory calculations for the TMP-F $\mathrm{F}_{4}$ TCNQ complex. The full potential local orbital (FPLO) basis set $^{21}$ with the generalized gradient approximation functional in its PBE form ${ }^{22}$ was used. We employed a
$10 \times 10 \times 10 k$ mesh and analyzed the band structure by fitting the bands near the Fermi level to a tight binding Hamiltonian ${ }^{23}$

$$
H=\sum_{i} \varepsilon_{i} c_{i}^{\dagger} c_{i}+\sum_{i j} t_{i j} c_{i}^{\dagger} c_{j}
$$

with the operators $c_{i}^{\dagger}\left(c_{i}\right)$ creating (annihilating) electrons at site $i$, onsite energies $\varepsilon_{i}$ and transfer integrals $t_{i j}$. We checked the uniqueness of the resulting fit using projective Wannier functions. ${ }^{24}$

We simulated uniaxial pressure along the stacking direction by scaling the lattice parameter $a$ of both TMP-TCNQ and TMP- $\mathrm{F}_{4}$ TCNQ in small steps and fully relaxing all internal coordinates using the projector augmented wave (PAW) basis and generalized gradient approximation functional as implemented in the Vienna ab initio simulation program (VASP). ${ }^{25,26}$ We used a $6 \times 6 \times 6 k$ mesh for TMP-TCNQ and a $4 \times 4 \times 4 k$ mesh for TMP-F ${ }_{4}$ TCNQ, with a plane wave cutoff of $500 \mathrm{eV}$.

\section{Results}

\subsection{Crystal structure}

The synthesized TMP-F $\mathrm{F}_{4}$ TCNQ crystals showed a triclinic structure with mixed stacks formed along their $a$-axis (see Table 1). Fig. 2 illustrates the packing arrangement from different perspectives.

All stacks have a common stacking axis. Along the stacks, TMP and $\mathrm{F}_{4}$ TCNQ lie almost perfectly on top of each other: Their respective planes (ignoring the methoxy groups) lie nearly perpendicular to the stacking direction while the geometrical centers of TMP and $\mathrm{F}_{4}$ TCNQ molecules are positioned on a common axis. The mean plane-to-plane separation is about $3.35 \AA$.

\subsection{Electrical conductivity}

Electrical conductivity measurements for one typical crystal are illustrated in Fig. 3. The crystal shown here had dimensions of roughly $360 \times 90 \times 50 \mu \mathrm{m}$. Current-temperature curves were recorded with an applied voltage of $100 \mathrm{~V}$, corresponding to a maximum electrical field of $11 \mathrm{kV} \mathrm{cm}{ }^{-1}$, for two distinct directions along the crystal, one parallel to its long axis and one perpendicular to it. The long axis corresponded to the stacking direction of the molecules ( $a$-axis). We note that the room temperature conductivity is more than one order of magnitude larger in the stacking direction than in perpendicular orientation.

Both $\sigma(T)$ characteristics show a thermally activated (Arrhenius) behavior but with different activation energies $E_{\mathrm{a}}$ (see Fig. 4). This behavior was only visible at $T>220 \mathrm{~K}$ as the resistance at lower temperatures became too high for the measurement setup. For current flow along the stacking axis a value of $E_{\mathrm{a}}=0.98 \mathrm{eV}$ was calculated based on the fit performed in Fig. 4. The perpendicular direction gave a value of $E_{\mathrm{a}}=0.84 \mathrm{eV}$.

\subsection{Degree of charge transfer}

In general, the fraction of charge ( $\rho$, in units of $e$ ) transferred from the donor to the acceptor (in the ground state) can be estimated by the frequency shift of the $\mathrm{C} \equiv \mathrm{N}$ stretching modes for which in most cases the shift grows linearly with the charge 
(a)

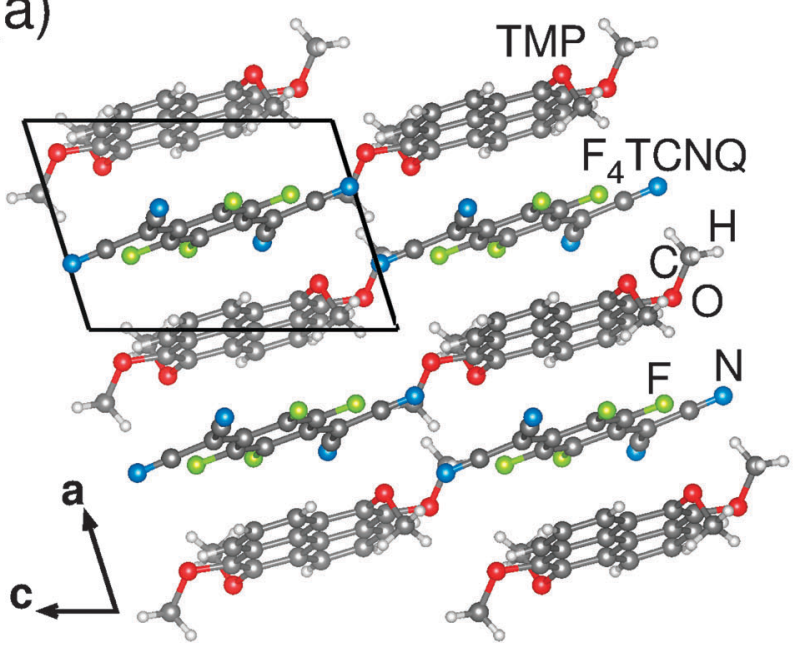

(b)

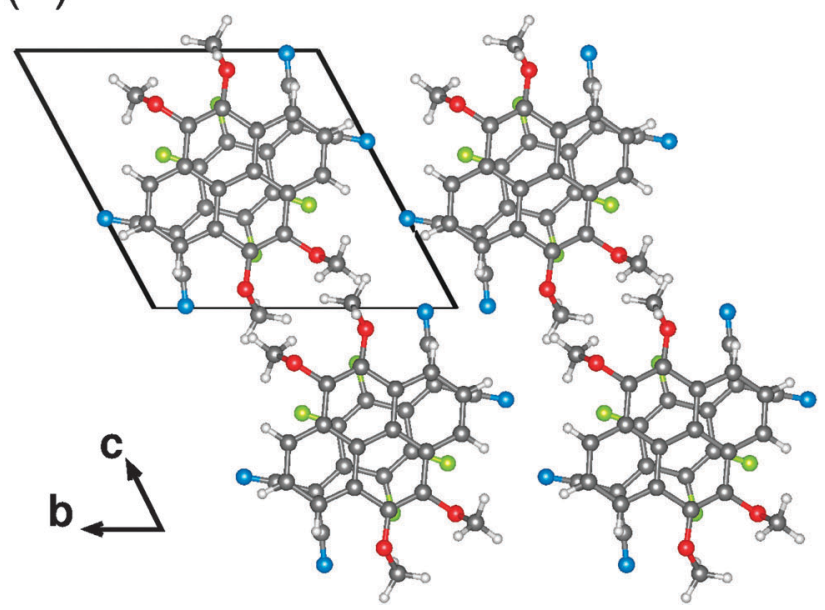

Fig. 2 Structure of TMP-F 4 TCNQ viewed along the (a) $b$ - and (b) a-axis

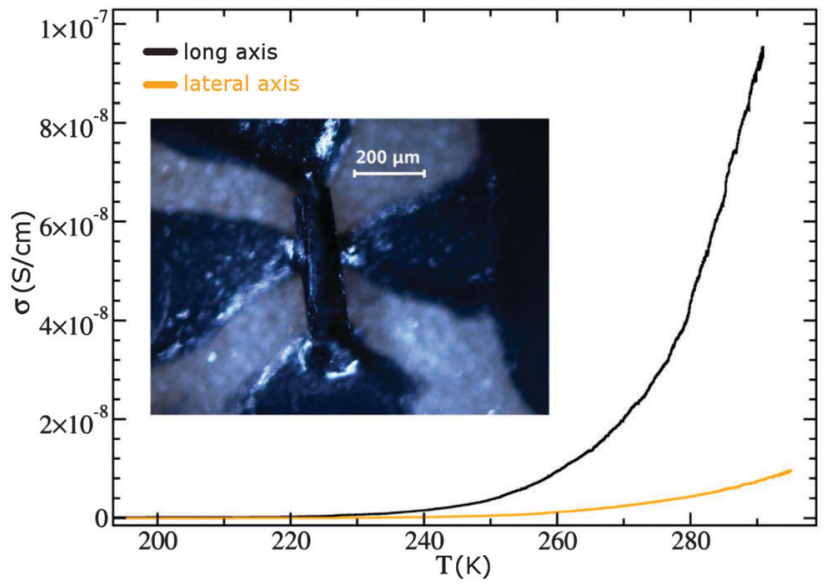

Fig. 3 Temperature-dependent conductivity measured on the TMP$\mathrm{F}_{4} \mathrm{TCNQ}$ crystal (shown with contacts in the optical micrograph (inset)) for two different directions.

transfer degree. ${ }^{27,28}$ The absorption of IR (infrared) radiation of isolated $\mathrm{F}_{4} \mathrm{TCNQ}$ was measured as a reference. The spectrum

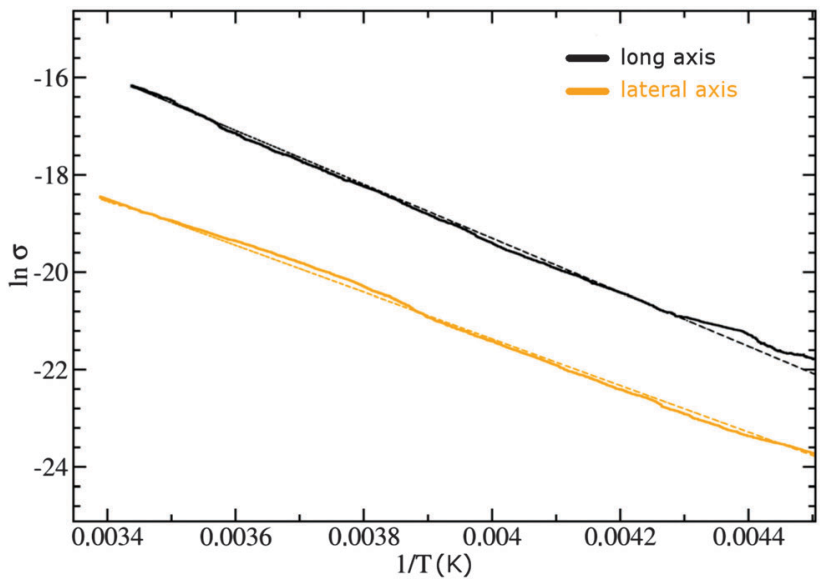

Fig. 4 Arrhenius plot and the corresponding linear fit (dashed lines) for both $\sigma(T)$ measurements.

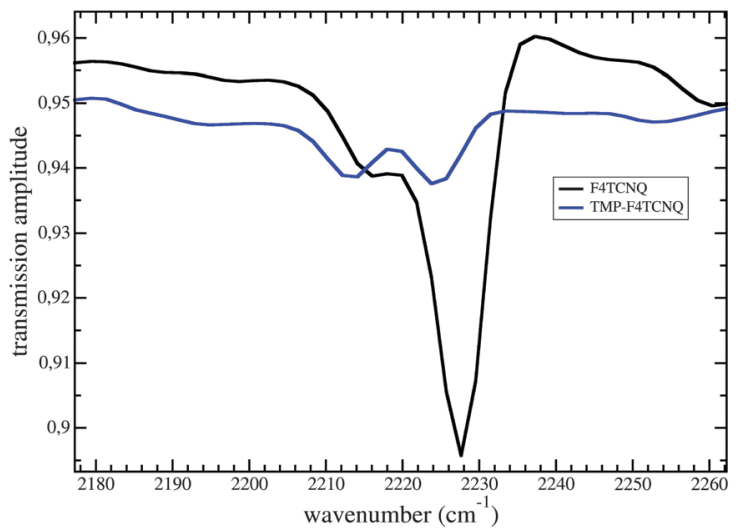

Fig. 5 IR absorption caused by the $\mathrm{CN}$ stretching vibrations in TMP$\mathrm{F}_{4} \mathrm{TCNQ}$ compared to that in $\mathrm{F}_{4} \mathrm{TCNQ}$. Both curves are an average over 16 scans.

shown in Fig. 5 illustrates IR bands at $2217 \mathrm{~cm}^{-1}$ and $2228 \mathrm{~cm}^{-1}$ corresponding to the modes $b_{2 u}$ and $b_{1 u}$.

Both values are in close agreement with the results from other measurements. ${ }^{29,30}$ The spectrum for $\mathrm{TMP}^{\mathrm{F}} \mathrm{F}_{4} \mathrm{TCNQ}$ shows two weak bands at $2213 \mathrm{~cm}^{-1}$ and $2224 \mathrm{~cm}^{-1}$ that probably correspond to the modes visible in the $\mathrm{F}_{4} \mathrm{TCNQ}$ spectrum being red-shifted by $3-4 \mathrm{~cm}^{-1}$ due to the charge transfer. Based on these numbers and on the $\nu$ values for fully ionized $\mathrm{F}_{4} \mathrm{TCNQ},{ }^{29} \rho$ can be calculated with a simple relationship given in ref. 27 . The results for the charge transfer degree are 0.12 in the case of $b_{1 u}$ and 0.09 for $b_{2 u}$.

\subsection{Charge transfer transition energy}

Fig. 6 shows two absorption peaks of TMP and $\mathrm{F}_{4} \mathrm{TCNQ}$ in solution in the region of interest: the left one at $592 \mathrm{~nm}$ corresponds to an energy of $2.1 \mathrm{eV}$, and the right one at $1132 \mathrm{~nm}$ corresponds to $1.1 \mathrm{eV}$. The latter presumably originates from the characteristic charge transfer transition which is typically associated with a broad peak shape like in our case.

The peak at $2.1 \mathrm{eV}$ could stem from intramolecular transitions in donor or acceptor (neutral or ionized) molecules that 


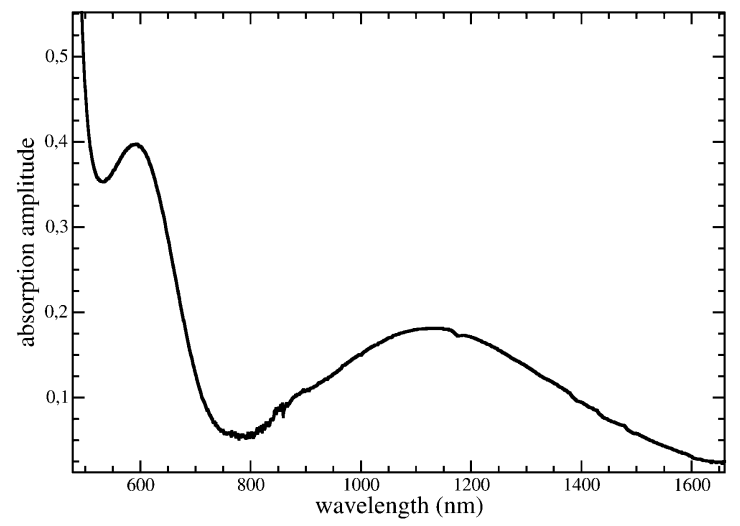

Fig. 6 Absorption spectrum of $\mathrm{TMP}-\mathrm{F}_{4} \mathrm{TCNQ}$. Data were recorded from partly reacted TMP and $\mathrm{F}_{4} \mathrm{TCNQ}$ in solution.

generally appear at higher energies than the CT transition. The same very likely applies to the strong absorption partly visible in the leftmost position of the figure. Here, one can refer to the measurements performed on neutral TMP (thin films) ${ }^{31}$ that showed an optical gap of $3.167 \mathrm{eV}(392.7 \mathrm{~nm})$. Similar peak energies were found for other donors/acceptors in the neutral or ionized state. ${ }^{18,31-33}$

\subsection{Electronic structure}

Fig. 7 shows the band structure of $\mathrm{TMP}-\mathrm{F}_{4} \mathrm{TCNQ}$ in a large energy window. Coordinates of the high symmetry points are $\Gamma=(0,0,0), X=(0.5,0,0), U=(0.5,0,0.5), R=(0.5,0.5,0.5)$, $V=(0.5,0.5,0), Y=(0,0.5,0), T=(0,0.5,0.5), Z=(0,0,0.5)$ in units of the reciprocal lattice vectors. The electronic structure for TMP- $\mathrm{F}_{4}$ TCNQ is calculated in a primitive cell containing one formula unit and it is characterized by well separated single bands of predominant TMP or $\mathrm{F}_{4} \mathrm{TCNQ}$ origin, respectively. These bands are a result of the hybridization between the highest occupied molecular orbital (HOMO) of TMP and the lowest unoccupied molecular orbital (LUMO) of $\mathrm{F}_{4} \mathrm{TCNQ}$ (see ref. 34 for details). The band below the Fermi level is mostly of TMP origin (donor) with some hybridization with $\mathrm{F}_{4} \mathrm{TCNQ}$ while the band above the Fermi level is of $\mathrm{F}_{4}$ TCNQ origin

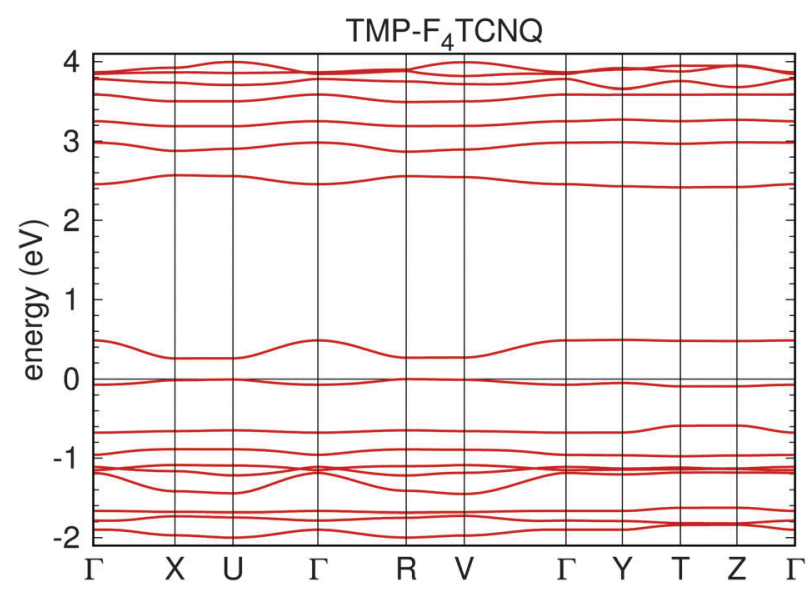

Fig. 7 Band structure of $\mathrm{TMP}-\mathrm{F}_{4} \mathrm{TCNQ}$ with a DFT gap of $E_{\mathrm{g}}=0.26 \mathrm{eV}$.

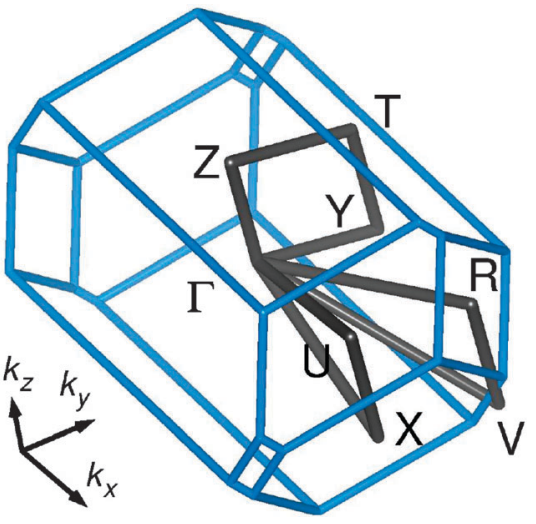

Fig. 8 Brillouin zone and the chosen $k$ path for triclinic TMP $-F_{4} T C N Q$.

(acceptor) with some hybridization with TMP. The bands are narrow and only show dispersion along paths where the $k_{x}$ coordinate is changing (for a visualization of the $k$ path, see Fig. 8). This indicates that the system is very one-dimensional along the a direction which is the stacking direction (compare Fig. 2). The GGA band gap is $E_{g}=0.26 \mathrm{eV}$. Correlation effects beyond the GGA approximation, like local electronic interactions $U$ or nonlocal Coulomb correlations $V$, have not been included in the calculations and could play a role in the estimation of the band gap. However, the DFT (GGA) results should be reliable in providing the trend that TMP- $\mathrm{F}_{4} \mathrm{TCNQ}$ has a narrower gap than TMP-TCNQ.

In Fig. 9 we show the density of states (DOS) for TMP$\mathrm{F}_{4} \mathrm{TCNQ}$. The effect of the charge transfer is seen in the lowest unoccupied states of $F_{4}$ TCNQ which show some degree of hybridization with the highest occupied states of TMP. Consequently, the highest occupied states of TMP- $\mathrm{F}_{4} \mathrm{TCNQ}$ have some admixture of $\mathrm{F}_{4} \mathrm{TCNQ} /$ cyano group character, and the lowest unoccupied states have contributions from TMP.

The charge transfer can be quantified using the charges on the atoms as obtained from the charge density; these numbers can be corroborated by integrating parts of the density of states

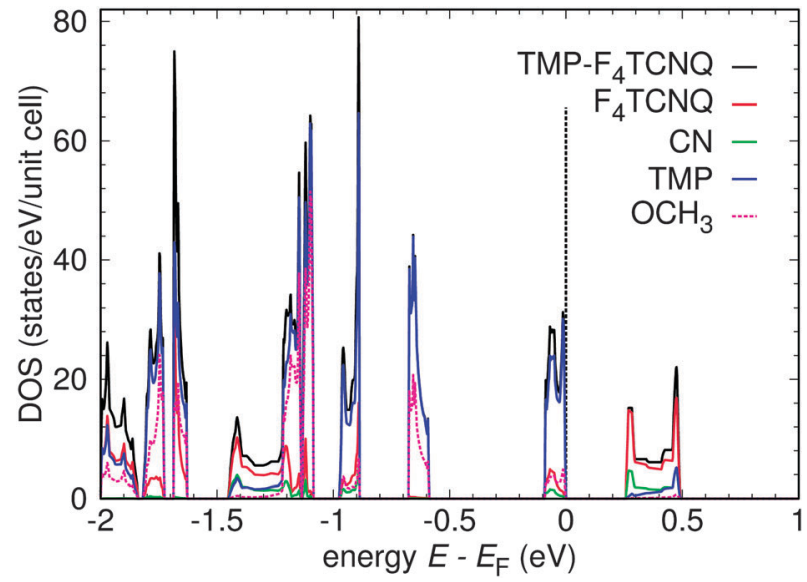

Fig. 9 Density of states of TMP- $\mathrm{F}_{4} \mathrm{TCNQ}$. The Fermi level is indicated by a dashed line. Contributions of TMP are shown in blue, of $F_{4} T C N Q$ in red, of the cyano groups in green and of the methoxy groups in purple. 
shown in Fig. 9. Note that both ways to determine the charge transfer involve different approximations and should not be expected to give exactly the same result. Analysis of the charge transfer yields a value of 0.20 electrons per formula unit from TMP to $\mathrm{F}_{4}$ TCNQ. This agrees roughly with 0.24 electrons $\mathrm{F}_{4}$ TCNQ-contribution to the highest occupied TMP-derived bands of TMP-F $F_{4}$ TCNQ (Fig. 9) and with 0.31 electrons TMP-contribution to the lowest unoccupied $\mathrm{F}_{4}$ TCNQ-derived bands. Comparison of this result with the charge transfer obtained from measurements (previous section) shows a disagreement that will be discussed below.

We now determine the tight-binding Hamiltonian (compare eqn (1)) for the highest occupied and lowest unoccupied bands of TMP-F 4 TCNQ. Fig. 10 shows perfect agreement of the obtained tight-binding bands with the DFT bands. The values of the tightbinding parameters obtained from projective Wannier functions are plotted in Fig. 11. The largest contributions in both valence and conduction bands correspond to nearest neighbor hoppings between alike molecules along the stacking direction $a$, which is related to the strong one-dimensional character of both bands.

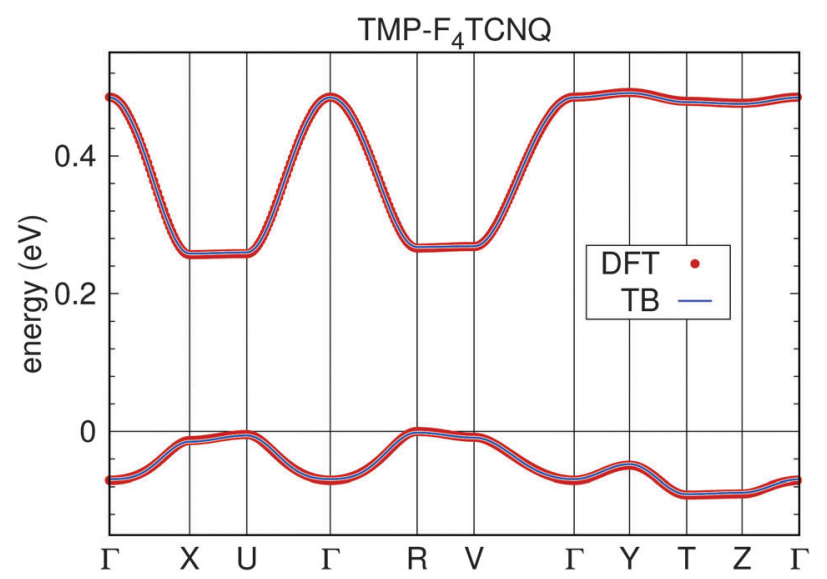

Fig. 10 Tight-binding bands for $\mathrm{TMP}-\mathrm{F}_{4} \mathrm{TCNQ}$ obtained from projective Wannier functions (solid line) shown together with the result of the DFT calculation.

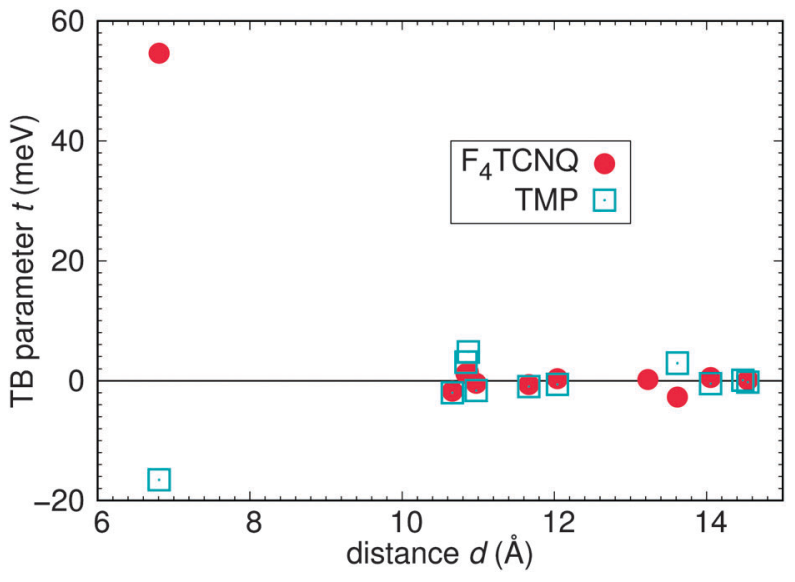

Fig. 11 Tight-binding parameters for TMP-F 4 TCNQ. No hoppings between TMP-derived and $\mathrm{F}_{4} \mathrm{TCNQ}$-derived bands are found.
Note that there are no tight-binding parameters connecting the two bands. This is a common feature in donor-acceptor systems.

\subsection{Uniaxial pressure simulation}

Eventually, it would be useful to develop methods to design charge transfer salts, relying not only on the quantum chemical analysis of binding energies for the donor and acceptor molecules, but taking into account also the arrangement of the molecules in a crystal. We now explore two aspects of the crystal structure that are important for the charge transfer: The spacing of the donor and acceptor molecules along the stacking axis and the respective arrangements of the charge distributions on the molecules. For that purpose, we fully relax within density functional theory with the GGA approximation the internal coordinates of the TMP-TCNQ and TMP-F 4 TCNQ structures for a small set of reduced lattice parameters $a$, thus simulating uniaxial pressure. Such an application of uniaxial pressure or, alternatively, biaxial tensile strain, as often observed in thin films, ${ }^{14}$ could in principle be performed also in experiments, but this is beyond the scope of the present investigations and in this work we rather consider these effects as a computer experiment that could allow us to predict whether application of pressure may induce small chemical modifications of donor or acceptor molecules that may be beneficial in the context of optimizing the charge transfer degree.

We consider here the consequences of uniaxial strain in TMP-F 4 TCNQ and also in TMP-TCNQ for comparison. Fig. 12 shows the evolution of the band gap, as well as the charge transfer from TMP to TCNQ in TMP-TCNQ and from TMP to $\mathrm{F}_{4} \mathrm{TCNQ}$ in TMP-F $\mathrm{F}_{4} \mathrm{TCNQ}$ as a function of the reduced lattice

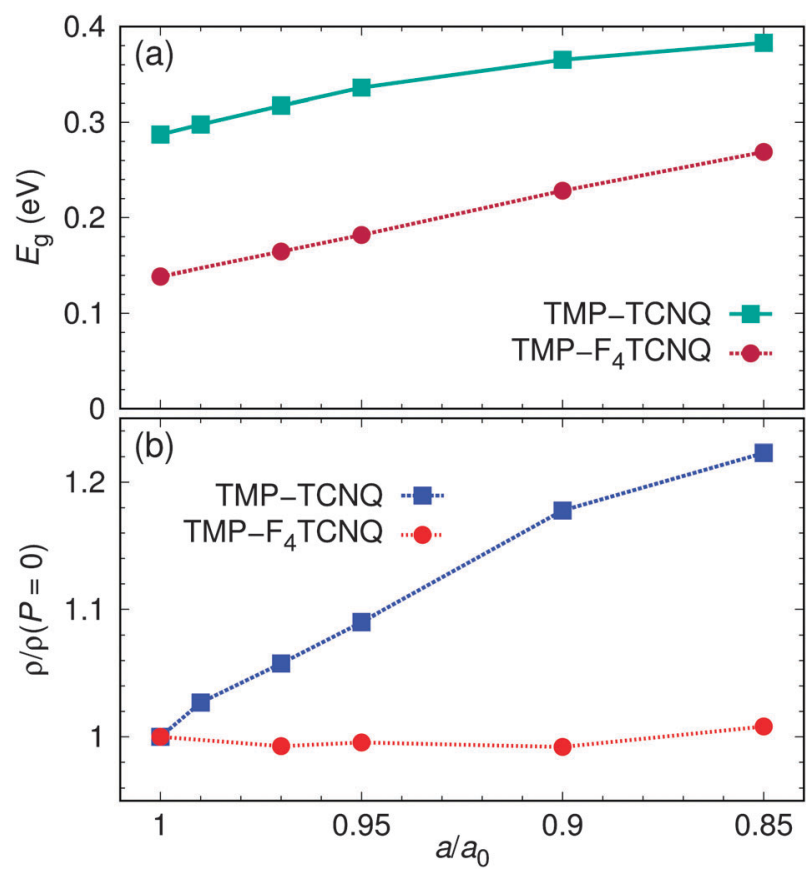

Fig. 12 Evolution of the band gap (a) and charge transfer (b) from TMP to $\mathrm{TCNQ}\left(\mathrm{F}_{4} \mathrm{TCNQ}\right)$ as a function of uniaxial compression along the stacking direction $a$. 
parameter $a$; $a_{0}=a(P=0)$ corresponds to the lattice parameter $a$ at ambient pressure. Note that the structures for different $a / a_{0}$ were obtained by relaxing the internal coordinates of the complexes within DFT. For TMP-TCNQ and $a / a_{0}=1$ the relaxed structure shows only small differences with respect to the experimental structure. For the calculated charge transfer in the relaxed structure we obtain an offset of about 0.2 with respect to the value deduced from the DFT calculations relying on the experimental crystal structure. For TMP-F ${ }_{4}$ TCNQ the corresponding offset is about 0.1 . In the representation of the uniaxial strain effects on the charge transfer, shown in Fig. 12(b), the results are normalized to the value calculated for the relaxed structures at zero strain. Interestingly, we find different trends in TMP-TCNQ and TMP-F 4 TCNQ: In TMPTCNQ, the charge transfer increases approximately linearly with compression along $a$, while in TMP- F $_{4}$ TCNQ it stays constant. For a more detailed analysis, we show in Fig. 13 the densities of states for all values of the $a / a_{0}$ ratio. As expected, the overall band width increases as a function of pressure in both cases. However, if we focus on the states directly below the Fermi level that correspond mostly to the highest occupied molecular orbitals of TMP, we see an important difference: While the dispersion corresponding to these states significantly increases with uniaxial compression in TMP-TCNQ, it remains nearly unchanged in $\mathrm{TMP}-\mathrm{F}_{4} \mathrm{TCNQ}$. In the next section we will

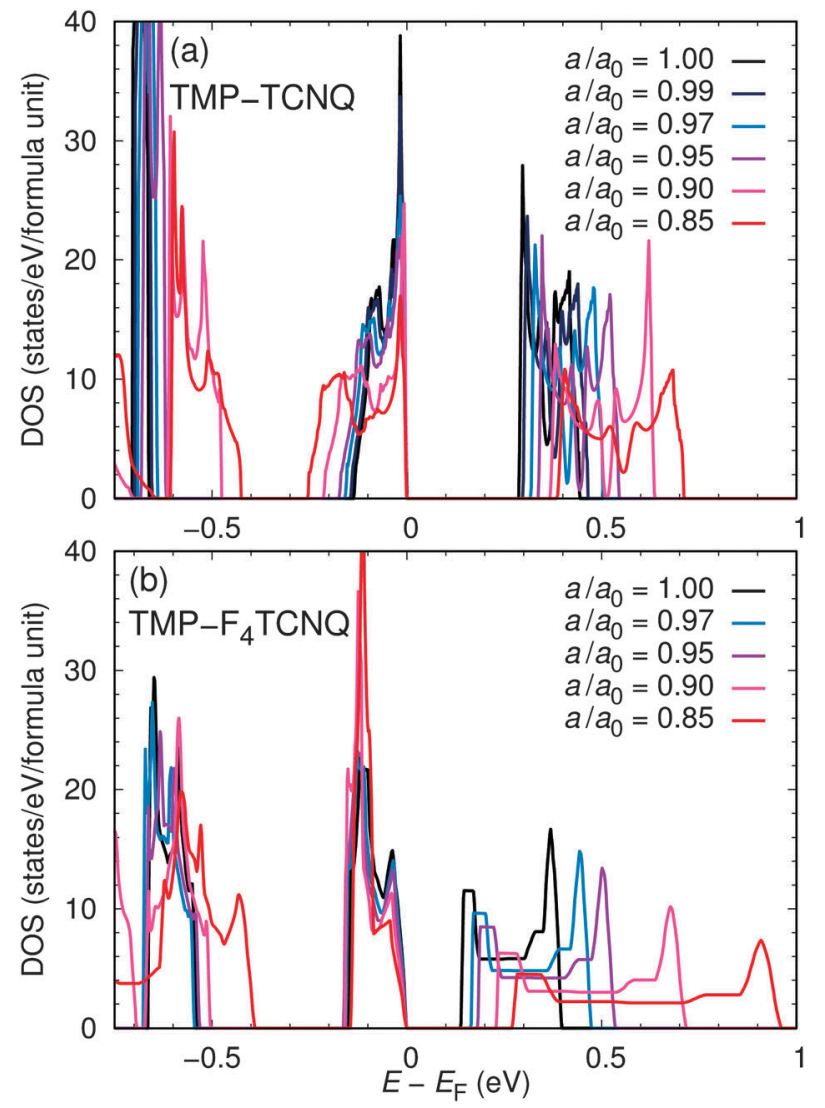

Fig. 13 Density of states (DOS) of (a) TMP-TCNQ and (b) TMP-F 4 TCNQ for different degrees of uniaxial compression along the stacking direction $a$. discuss possible reasons for this different behavior related to the differences in the charge density arrangement of the two compounds.

\section{Discussion}

The crystal structure of TMP- $\mathrm{F}_{4}$ TCNQ with its simple and clear stacking arrangement of donor-acceptor molecules gives rise to a markedly reduced dimensionality of its electronic transport characteristics. This is confirmed by both our conductivity measurements and band structure calculations. The dispersion visible only along $k_{x} \| a$ is reflected in an appreciable increase of the conductivity along the stacking axis. This one-dimensional character is a common phenomenon in CT systems but not necessarily pronounced in mixed-stack CT systems. However, we observe that an almost perfect one-dimensional geometry does not necessarily lead to an optimized interaction that promotes maximum conductivity. The semiconductor-like behavior of $\sigma(T)$ below room temperature in TMP-TCNQ is pronounced due to the rather high activation energies. The conductivity is further limited by the rather small charge transfer, if one takes into account that high $\sigma$-values should not be expected for $\rho<0.4 .^{35}$

In order to further analyze the influence of the geometry of the stacking on the conductivity behavior as well as the role of the specific acceptor $\mathrm{F}_{4}$ TCNQ in more detail, we compare in this section the electronic properties of TMP-F $\mathrm{F}_{4} \mathrm{TCNQ}$ and TMP-TCNQ. ${ }^{31,34}$

TMP-TCNQ shows a mixed-stack arrangement like TMP$\mathrm{F}_{4}$ TCNQ but the low dimensionality is far less pronounced. ${ }^{34,36}$ TMP and TCNQ molecules are tilted against the stacking axis by slightly different angles, i.e. their planes are less parallel compared to the TMP- $\mathrm{F}_{4}$ TCNQ geometry. Within the stacks, they are shifted along $b$ and $c$ so that the geometrical centers of adjacent molecules do not coincide when one plane is projected onto another. This shift becomes particularly apparent when comparing the distribution of excess charges viewed along the stacking axis, as shown in Fig. 14. By introducing the fluor in $\mathrm{F}_{4}$ TCNQ a significant increase of negative excess charge at the acceptor ring position occurs which seems to act as a strong docking point for the positive excess charge located at the ring close to the methoxy groups of the donor. This leads to a ringcentering effect in the donor-acceptor stacking of TMP- $\mathrm{F}_{4} \mathrm{TCNQ}$ which also has the consequence of improving the planarity of the $\mathrm{F}_{4}$ TCNQ molecule. In TMP-TCNQ the planarity of the TCNQ structure is reduced when compared with $\mathrm{F}_{4} \mathrm{TCNQ}$ as the CN groups show stronger twists with respect to the central ring and even the ring itself is not really planar. From these observations we draw two conclusions relating to the molecular overlap and the associated conductivity, as well as the expected behavior under applied uniaxial pressure along the stacking axis.

First, planarity is generally regarded as a relevant factor for higher conductivity due to the increase of the molecular orbital overlap integrals. ${ }^{8}$ The reduced planarity and lateral offset in the ring stacking in TMP-TCNQ are therefore reflected by 
(a) $\mathrm{F}_{4} \mathrm{TCNQ}$

(d) TMP-F $-\mathrm{F}_{4} \mathrm{TCNQ}$

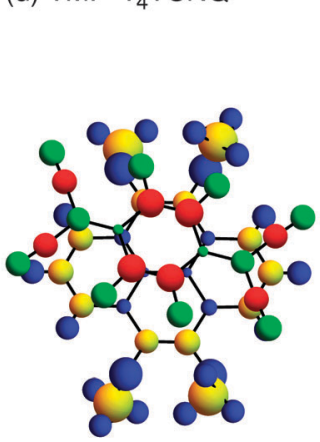

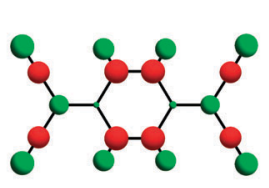

(b) TMPe

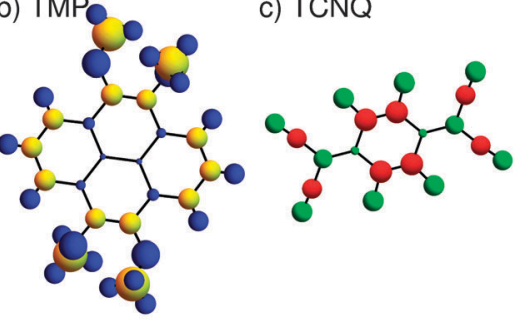

(e) TMP-TCNQ

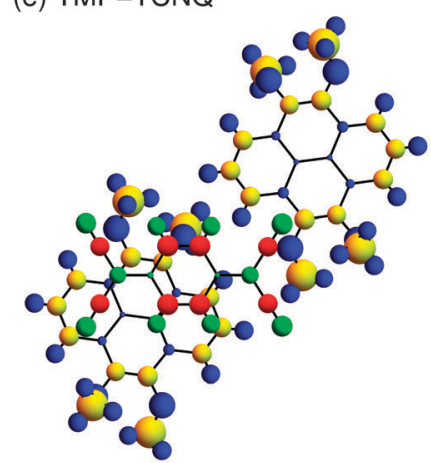

Fig. 14 Distribution of charges in (a) $F_{4} T C N Q$, (b) TMP, (c) TCNQ, (d) TMP-F $F_{4} T C N Q$ and (e) TMP-TCNQ. Spheres are drawn with the volume proportional to the excess charge (positive in blue and green and negative in yellow and red) on each atom. In (d) and (e), the actual alignment of the donor and acceptor from the crystal structure is shown.

significantly reduced conductivity values and the absence of an observable anisotropy when compared to TMP-F $\mathrm{F}_{4}$ TCNQ. In fact, the exact room-temperature value(s) of the conductivity for different directions of a mm-sized TMP-TCNQ single crystal could not be determined as its resistance exceeded the isolation resistance of our setup. Nevertheless, we can state an upper limit of about $10^{-11} \mathrm{~S} \mathrm{~cm}^{-1}$. The fundamental differences between TMP-TCNQ and TMP- $\mathrm{F}_{4}$ TCNQ are confirmed by the band structure calculations. TMP-TCNQ bands are narrower and show weak dispersion in all directions. The bandwidth is only $0.1 \mathrm{eV}$ while it is $0.25 \mathrm{eV}$ for TMP-F ${ }_{4}$ TCNQ (see Fig. 15).

Second, with regard to the expected behavior of the charge transfer degree under uniaxial compression, the near-ideal planarity

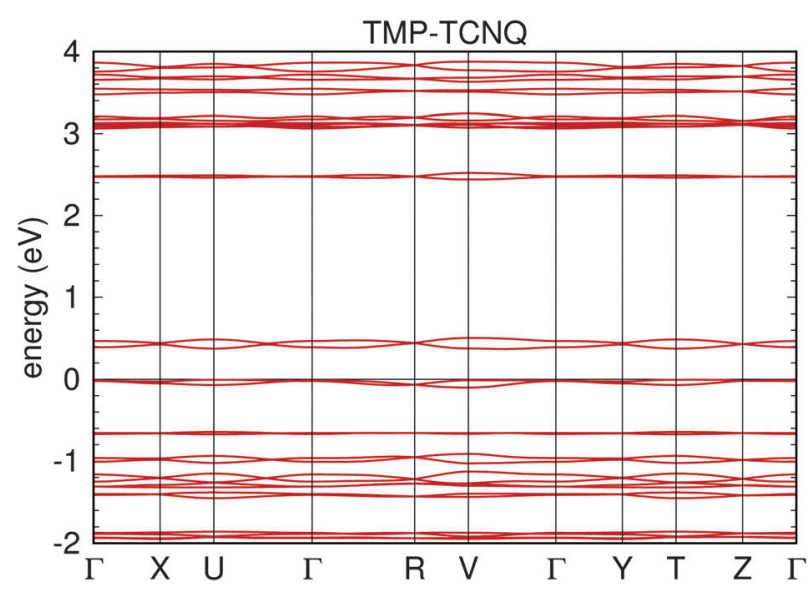

Fig. 15 Band structure of TMP-TCNQ with a DFT gap of $E_{\mathrm{g}}=0.37 \mathrm{eV}$. and ring-stacking of TMP- $\mathrm{F}_{4}$ TCNQ seem to preclude a significant increase of the donor-acceptor hybridization, as clearly suggested by the results of the DFT calculations shown in Fig. 12. For TMP-TCNQ, on the other hand, a significant increase of the planarity of the acceptor molecules under uniaxial pressure may be expected, as well as possible lateral rearrangements leading to improved ring stacking. In this case, a clear tendency for a growing charge transfer degree should be expected, as strongly suggested by the DFT calculations. We consider these predictions to be a robust feature despite the fact that the calculated degrees of charge transfer, in particular for the fully relaxed structures, do not agree very well with the experimental values obtained from analyzing the $\mathrm{CN}$ vibration frequency shifts. We conclude this section with some comments in this direction.

Regarding the degree of charge transfer in TMP-TCNQ the $\mathrm{CN}$ vibration showed a frequency shift from $2227 \mathrm{~cm}^{-1}$ (pure TCNQ) to $2221 \mathrm{~cm}^{-1}$ indicating a charge transfer of 0.14 . In this case, the theoretical analysis of charges, relying on the experimental crystal structure, produced a nearly perfect agreement as an overall charge transfer of 0.13 from TMP to TCNQ was found. It agrees roughly with 0.16 electrons TCNQ contribution to the highest occupied TMP-derived bands of TMP-TCNQ, and with 0.21 electrons TMP contribution to the lowest unoccupied TCNQ-derived bands (experimental structure). In the case of TMP- $\mathrm{F}_{4}$ TCNQ the agreement between theoretical ( $c$ a. 0.2-0.3) and the extracted values from infrared measurements $(0.12)$ is not as good. Considering that the absolute $\mathrm{CN}$ vibration frequency shifts are rather small, the error margins for the experimentally deduced charge transfer values are about 0.05. In addition, the observed changes in $\rho$ derived from the DFT calculations when allowing for full relaxation, in particular for TMP-TCNQ, indicate that already subtle changes in the crystal structure can lead to substantial changes. Taking this together, the deduced absolute values for the charge transfer degree in the TMP-TCNQ and TMP-F ${ }_{4}$ TCNQ systems have to be considered with caution. However, the predicted uniaxial pressure trends and their rationalization with a view to the stacking arrangement and excess charge distributions provide valuable insight into the somewhat counter-intuitive trends observed when going from TMP-TCNQ to TMP-F ${ }_{4}$ TCNQ. This work has shown that simple arguments that the significantly larger electron affinity of F $_{4}$ TCNQ ( ca. $5.2 \mathrm{eV}$ ) in comparison to that of TCNQ $(c a .4 .8 \mathrm{eV})^{8}$ should lead to an increased charge transfer do not hold. The present comparison also forms an interesting analogy to similar investigations like the one by Torrance, ${ }^{37}$ who contrasted HMTTF-TCNQ with HMTTF-F 4 TCNQ. In their study the different acceptors led to isostructural CT systems but with very different degrees of charge transfer. As a result HMTTF-F $\mathrm{F}_{4}$ TCNQ becomes a Mott insulator while HMTTF-TCNQ is an organic metal.

\section{Conclusion}

We have investigated the structural and electronic transport properties of the new charge transfer compound TMP- $\mathrm{F}_{4} \mathrm{TCNQ}$ 
in the form of single crystals. Both crystal and band structures show a pronounced reduction of dimensionality as the nearly perfect geometrical overlap of molecules along the mixed donoracceptor stacks leads to a one-dimensional transport characteristics. The predictions are qualitatively confirmed by the measurement of anisotropic, semiconductor-like electrical conductivity that is largest in the stacking direction. Based on IR spectroscopy a small charge transfer of about 0.1 was found while theoretical values as well as some general considerations concerning the maximization of charge transfer suggest a higher value. In comparison, the related CT complex TMP-TCNQ, introduced in recent publications, ${ }^{31,34}$ shows clear structural differences. They result in a more isotropic transport behaviour with a significant overall reduction of electrical conductivity owing to the loss of the apparently optimized molecular overlap found in TMP-F $\mathrm{F}_{4}$ TCNQ. At the same time our theoretical study on the evolution of the charge transfer degree in TMP- $\left(\mathrm{F}_{4}\right) \mathrm{TCNQ}$ suggests that for TMP-TCNQ a significant increase under uniaxial pressure along the stacking axis should occur whereas no

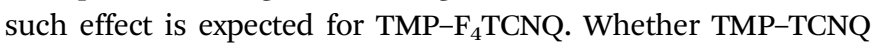
may be a candidate for a pressure-induced neutral-ionic transition remains for future resolution.

\section{Acknowledgements}

We thank V. Solovyeva, R. Rommel, M. Schmidt and S. Bekö for experimental support as well as helpful discussions. This work was funded through SFB/TR 49 by the Deutsche Forschungsgemeinschaft.

\section{References}

1 S. Liu, J. Shi, E. W. Forsythe, S. M. Blomquista and D. Chiu, Synth. Met., 2009, 159, 1438-1442.

2 K. Shibata, H. Wada, K. Ishikawa, H. Takezoe and T. Mori, Appl. Phys. Lett., 2007, 90, 193509.

3 S. Horiuchi, T. Hasegawa and Y. Tokura, J. Phys. Soc. Jpn., 2006, 75, 051016.

4 T. Mori, J. Phys.: Condens. Matter, 2008, 20, 184010.

5 Y. Takahashi, T. Hasegawa, Y. Abe, Y. Tokura, K. Nishimura and G. Saito, Appl. Phys. Lett., 2005, 86, 063504.

6 Y. Takahashi, T. Hasegawa, Y. Abe, Y. Tokura and G. Saito, Appl. Phys. Lett., 2006, 88, 073504.

7 S. Roth and D. Carroll, One-Dimensional Metals, Wiley-VCH, 2004.

8 G. Saito and Y. Yoshida, Bull. Chem. Soc. Jpn., 2007, 80, $1-137$.

9 S. Horiuchi and Y. Tokura, Nat. Mater., 2008, 7, 357-366.

10 L. Zhu, Y. Yi, Y. Li, E.-G. Kim, V. Coropceanu and J.-L. Brédas, J. Am. Chem. Soc., 2012, 134, 2340-2347.

11 I. Shokaryev, A. J. C. Buurma, O. D. Jurchescu, M. A. Uijttewaal, G. A. de Wijs, T. T. M. Palstra and R. A. de Groot, J. Phys. Chem. A, 2008, 112, 2497-2502.

12 S. Horiuchi, R. Kumai, Y. Okimoto and Y. Tokura, Chem. Phys., 2006, 325, 78-91.
13 M. H. Lemé-Cailleau, M. L. Cointe, H. Cailleau, T. Luty, F. Moussa, J. Roos, D. Brinkmann, B. T. C. Ayache and N. Karl, Phys. Rev. Lett., 1997, 79, 1690.

14 M. Huth, A. Rippert, R. Sachser and L. Keller, Mater. Res. Express, 2014, 1, 046303.

15 V. Solovyeva and M. Huth, Synth. Met., 2011, 161, 976.

16 V. Solovyeva, K. Keller and M. Huth, Thin Solid Films, 2009, 517, 6671.

17 M. Filatov, Phys. Chem. Chem. Phys., 2013, 13, 144.

18 J. B. Torrance, J. E. Vazquez, J. J. Mayerle and V. Y. Lee, Phys. Rev. Lett., 1981, 46, 253-257.

19 G. M. Sheldrick, Acta Crystallogr., Sect. A: Cryst. Phys., Diffr., Theor. Gen. Crystallogr., 2008, 64, 112-122.

20 Publication No. CCDC 1002047.

21 K. Koepernik and H. Eschrig, Phys. Rev. B: Condens. Matter Mater. Phys., 1999, 59, 1743.

22 J. P. Perdew, K. Burke and M. Ernzerhof, Phys. Rev. Lett., 1996, 77, 3865.

23 H. C. Kandpal, I. Opahle, Y.-Z. Zhang, H. O. Jeschke and R. Valent, Phys. Rev. Lett., 2009, 103, 067004.

24 A. C. Jacko, H. Feldner, E. Rose, F. Lissner, M. Dressel, R. Valent and H. O. Jeschke, Phys. Rev. B: Condens. Matter Mater. Phys., 2013, 87, 155139.

25 G. Kresse and J. Hafner, Phys. Rev. B: Condens. Matter Mater. Phys., 1993, 47, R558.

26 G. Kresse and J. Furthmüller, Phys. Rev. B: Condens. Matter Mater. Phys., 1996, 54, 11169.

27 A. Salmerón-Valverde, J. G. Robles-Martnez and A. Zehe, Cryst. Res. Technol., 1994, 29, 703-706.

28 J. S. Chappell, A. N. Bloch, W. A. Bryden, M. Maxfield, T. O. Poehler and D. O. Cowan, J. Am. Chem. Soc., 1981, 103, 2442-2443.

29 M. Meneghetti and C. Pecile, J. Chem. Phys., 1986, 84, 4149-4162.

30 P. Pingel, L. Zhu, K. S. Park, J.-O. Vogel, S. Janietz, E.-G. Kim, J. P. Rabe, J.-L. Brédas and N. Koch, J. Phys. Chem. Lett., 2010, 1, 2037-2041.

31 K. Medjanik, S. Perkert, S. Naghavi, M. Rudloff, V. Solovyeva, D. Chercka, M. Huth, S. A. Nepijko, T. Methfessel, C. Felser, M. Baumgarten, K. Müllen, H.-J. Elmers and G. Schönhense, Phys. Rev. B: Condens. Matter Mater. Phys., 2010, 82, 245419.

32 J. B. Torrance, B. A. Scott, B. Welber, F. B. Kaufman and P. E. Seiden, Phys. Rev. B: Condens. Matter Mater. Phys., 1979, 19, 730-741.

33 G. Qian, B. Dai, M. Luo, D. Yu, J. Zhan, Z. Zhang, D. Ma and Z. Y. Wang, Chem. Mater., 2008, 20, 6208-6216.

34 K. Medjanik, D. Chercka, P. Nagel, M. Merz, S. Schuppler, M. Baumgarten, K. Müllen, S. A. Nepijko, H.-J. Elmers, G. Schönhense, H. O. Jeschke and R. Valent, J. Am. Chem. Soc., 2012, 134, 4694-4699.

35 A. Salmerón-Valverde, J. G. Robles-Martnez, J. Garca-Serrano, R. Gómez, R. M. Ridaura, M. Quintana and A. Zehe, Mol. Eng., 1999, 8, 419-426.

36 S. Kawano, M. Baumgarten, D. Chercka, V. Enkelmann and K. Müllen, Chem. Commun., 2013, 49, 5058.

37 J. B. Torrance, J. J. Mayerle, K. Bechgaard, B. D. Silverman and Y. Tomkiewicz, Phys. Rev. B: Condens. Matter Mater. Phys., 1980, 22, 4960-4965. 